\title{
Triclosan produces statistically significant reduction in plaque, gingivitis and caries but not clinically important benefit
}

\author{
Abstracted from \\ Riley P, Lamont T. \\ Triclosan/copolymer containing toothpastes for oral health. \\ Cochrane Database Syst Rev 2013; 12: Art. No.: CD010514. DOI: 10.1002/14651858.CD010514.pub2. \\ Address for correspondence: Luisa Fernandez Mauleffinch, Review Group Co-ordinator, Cochrane Oral Health Group, MANDEC, School of \\ Dentistry, University of Manchester, Higher Cambridge Street, Manchester, M15 6FH, UK. E-mail: luisa.fernandez@manchester.ac.uk
}

\section{Question: Are triclosan/copolymer containing fluoride toothpastes more effective than fluoride toothpastes for control of caries, plaque and gingivitis in children and adults?}

Data sources The Cochrane Oral Health Group's Trials Register, the Cochrane Central Register of Controlled Trials (CENTRAL), Medline, Embase and the US National Institutes of Health Trials Register. Study selection Randomised controlled trials (RCTs) of parallel or crossover design, (with a sufficient wash-out period) of at least six months duration, and irrespective of language or publication status. Data extraction and synthesis Study assessment and data extraction were carried out independently by at least two reviewers. Metaanalysis was conducted using random-effects models when there were at least four studies (fixed-effect models when fewer than four studies), reporting mean differences (MD) for continuous data and risk ratios (RR) for dichotomous data.

Results Thirty studies involving 14,835 patients were included. Ten studies were considered to be a low risk of bias, nine at high risk and the remaining 11 were assessed as at unclear risk. After six to seven months use of triclosan/copolymer toothpaste there was a statistically significant reduction in plaque in favour of triclosan/copolymer of 22\% (20 studies, 2675 patients, moderate-quality evidence).

There was also a statistically significant reduction in plaque severity of $41 \%$ (13 studies, 1850 patients, moderate-quality evidence). Triclosan/copolymer toothpaste also reduced gingival inflammation statistically significantly by $22 \%$ after six to nine months of use (20 studies, 2743 patients, moderate-quality evidence). After 36 months of use there was no evidence of a difference between triclosan/copolymer toothpaste and control in the development of periodontitis (attachment loss) (RR $0.92,95 \% \mathrm{Cl} 0.67$ to 1.27 , one

This paper is based on a Cochrane Review published in the Cochrane Library 2013, issue 12 (see www.thecochranelibrary.com for information). Cochrane Reviews are regularly updated as new evidence emerges and in response to feedback, and the Cochrane Library should be consulted for the most recent version of the review. study, 480 patients, low-quality evidence). After 24 to 36 months of use, triclosan/copolymer toothpaste slightly reduced coronal caries by about 5\% (four studies, 9692 patients, high-quality evidence). One study (1357 patients, moderate-quality evidence) suggested that after 36 months of use triclosan/copolymer toothpaste probably reduced root caries.

After six months of use triclosan/copolymer toothpaste may have reduced the mean total calculus by about $15 \%$. There were no data available for meta-analysis regarding adverse effects, but 22 studies (73\%) reported that there were no adverse effects caused by either the experimental or control toothpaste.

There was considerable heterogeneity present in the meta-analyses for plaque, gingivitis and calculus. Plaque and gingivitis showed such consistent results that it did not affect our conclusions, but the reader may wish to interpret the results with more caution.

Conclusions There was moderate-quality evidence showing that toothpastes containing triclosan/copolymer, in addition to fluoride, reduced plaque, gingival inflammation and gingival bleeding when compared with fluoride toothpastes without triclosan/copolymer. These reductions may or may not be clinically important, and are evident regardless of initial plaque and gingivitis levels, or whether a baseline oral prophylaxis had taken place or not.

High-quality evidence showed that triclosan/copolymer toothpastes led to a small reduction in coronal caries. There was weaker evidence to show that triclosan/copolymer toothpastes may have reduced root caries and calculus, but insufficient evidence to show whether or not they prevented periodontitis. There do not appear to be any serious safety concerns regarding the use of triclosan/copolymer toothpastes in studies up to three years in duration.

\section{Commentary}

Microbial biofilms, such as dental plaque, represent the primary cause of diseases of bacterial origin, including dental caries and periodontal disease. Dental biofilms are not easily controlled by mechanical means and therefore we must prevent dental disease by preventing the formation of, or disrupting biofilms in the oral cavity. 1 Tooth brushing is the most universally performed homecare intervention in the prevention of dental disease. It serves to both mechanically dis- 
rupt dental plaque, while also serving as a means to deliver various antimicrobial agents to the oral cavity. The standard practice of using fluoridated toothpaste has led to a significant reduction in the prevalence of dental caries in western civilisations; however, it has contributed very little to the reduction of periodontal diseases. ${ }^{2}$

Triclosan is a broad-spectrum antibacterial agent with low toxicity. When combined with a copolymer for substantively, it can be added to toothpastes to reduce plaque and gingival inflammation, a necessary precursor to the development of more severe periodontal disease. ${ }^{1}, 3$ While chlorhexidine may have a greater antimicrobial effect, triclosan is more compatible with other toothpaste ingredients. In addition, triclosan does not stain teeth or have an unpleasant taste, two common disadvantages associated with the use of chlorhexidine. ${ }^{2}$

Riley and Lamont used the GRADE criteria to conduct a rigorous systematic review, analysing and reporting findings of nearly 15,000 patients from 30 studies. All randomised controlled trials (RCTs) of parallel or crossover design, irrespective of language or publication status were included. Studies were of sufficient time - a minimum of six months for assessment of gingival health and a minimum of two years to assess dental caries. The length of study time was also necessary to eliminate the Hawthorne effect and to assess the long-term side effects of triclosan.

The authors were very clear on defining their inclusion criteria, which comprised the types of studies, participants, interventions and outcomes that would be included in their review. Primary (gingival health and plaque scores) and secondary outcomes (caries and calculus) were all measured using validated indices. In addition, the authors considered patient-centred outcomes, although none was available, and adverse effects, which are important when considering the use of the product orally. Any conditions that may have confounded the outcomes were excluded, including medical conditions, orthodontic appliances, pre-existing periodontitis and supervised brushing.

The review authors independently assessed the risk of bias of all included studies using the Cochrane Collaboration's domainbased, two-part tool. This instrument evaluates seven domains of risk of bias. They attempted to contact study authors for missing information and clarification, particularly with concerns of how the studies were conducted (for example, blinding). Ten studies were considered at low risk of bias, nine at high risk and eleven at unclear risk of bias. Labelling studies for unclear risk of bias occurred if the authors could not ascertain the appropriate information from the authors. The designation of high risk of bias was awarded because of lack of blinding in one study, incomplete outcome data in two studies, and selective reporting of data in seven studies.

Only three studies mentioned sample size calculations, with only one definitively meeting the target. Three studies included in the review were potentially truly independent, whereas 27 studies were in one way or another associated with industry, mainly through support and authorship. The authors conducted meta-analyses using random-effects models for four or more studies and fixed-effects models for fewer than four studies. They reported mean differences (MD) for continuous data and risk ratios (RR) for dichotomous data. Heterogeneity was assessed using forest plots and statistically using a $\mathrm{Chi}^{2}$ test. The authors also carried out subgroup analyses and sen- sitivity analysis to ensure robust conclusions by excluding studies of high or unclear risk of bias.

The results of this Cochrane review are nearly identical to those of a similar review from a decade ago. ${ }^{4}$ Fifteen additional studies were used in the current analysis; this adds to the certainty of results found. Statistically significant results were found for most outcomes, some with impressive sounding reductions in outcomes (for example, $48 \%$ reduction in bleeding in the intervention group as compared to the control group). However, one must consider the clinical importance of such findings. The above example represents a mere reduction of 0.13 , with the control group mean scoring 0.27 on a $0-3$ scale. When the numbers are truly dissected, this is hardly anything to get excited about. All the outcomes show similar reductions.

While the outcomes may not be overwhelmingly remarkable from a clinical standpoint, the volume of moderate to high quality evidence supports that triclosan improves oral health outcomes. When compared to fluoride only toothpaste, toothpaste containing fluoride and triclosan/copolymer reduced plaque, gingival inflammation and gingival bleeding. High quality evidence shows a small reduction in coronal caries. Weaker evidence indicates reduced root caries and calculus can be expected, but there was insufficient evidence to show any prevention of periodontitis. Although triclosan appears to aid in the reduction of plaque and gingival inflammation, precursors to periodontitis, we need additional long-term studies to assess this outcome fully. There was considerable heterogeneity in the meta-analyses for plaque, gingivitis and calculus, but the authors considered this to be inconsequential for their conclusions on plaque and gingivitis given the large amount of consistent data included in the review.

There do not appear to be any serious safety concerns regarding the use of triclosan/copolymer toothpastes in studies up to three years in duration. There were no data available for the meta-analysis of adverse effects, with 22 studies explicitly reporting no adverse effects and seven groups reporting no information in this regard whatsoever. One study reported a mild adverse effect of tooth staining, but it was unclear which group had experienced the effect and whether or not the staining was attributable to triclosan/copolymer or another antiplaque/antigingivitis agent.

\section{Practice points}

- The volume of evidence, and its reasonable quality, has provided clear evidence of the modest benefits of using a triclosan/ copolymer toothpaste

- Tooth brushing with such toothpaste is a relatively inexpensive intervention that can be carried out by the vast majority of people in a domestic setting.

\footnotetext{
Ferne Kraglund

Department of Dental Clinical Sciences, Dalhousie University, Halifax, Nova Scotia, Canada

1. Newman MG, Takei H, Klokkevold PR, Carranza FA. Carranza's Clinical Periodontology. 10th ed. W.B. Saunders Co Ltd, 2006.

2. Blinkhorn A, Bartold PM, Cullinan MP, et al. Is there a role for triclosan/copolymer toothpaste in the management of periodontal disease? Br Dent / 2009; 207: 117-125.

3. Gunsolley JC. A meta-analysis of six-month studies of antiplaque and antigingivitis agents. J Am Dent Assoc 2006; 137: 1649-1657.

4. Davies RM, Ellwood RP, Davies GM. The effectiveness of toothpaste containing triclosan and polyvinyl-methyl ether maleic acid copolymer in improving plaque control and gingival health: a systematic review. J Clin Periodontol 2004; 31: 1029-1033.

Evidence-Based Dentistry (2014) 15, 6-7. doi: 10.1038/sj.ebd.6400980
} 